\title{
Three-Dimensional FDTD Modeling of a GPR
}

\author{
UĞUR OĞUZ AND LEvent GüreL* \\ DEPARTMENT OF ELECTRICAL AND ELECTRONICS ENGINEERING \\ BILKENT UNIVERSITY \\ BILKENT, ANKARA, TURKEY \\ (lgurelQee.bilkent.edu.tr)
}

\section{Introduction}

Numerical modeling and simulation of ground-penetrating radar (GPR) systems have been recognized as the preferred means of obtaining an understanding of subsurfacescattering mechanisms. Among the techniques used, the finite-difference time-domain (FDTD) method has been distinctly popular [1]-[5] due to its versatility in solving problems involving arbitrarily complicated inhomogeneities. In this paper, realistic threedimensional GPR scenarios are simulated using the FDTD method and the perfectlymatched layer (PML) $[6,7]$ absorbing boundary conditions. The radar unit, which contains the transmitting and receiving antennas, moves over the ground-air interface on a predetermined path.

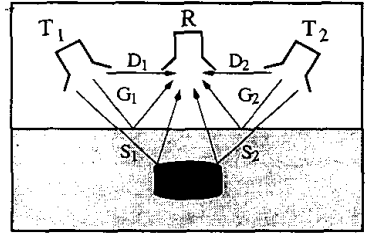

(a)

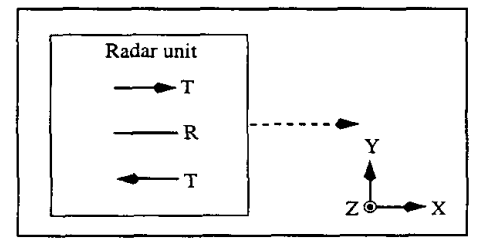

(b)

Figure 1: (a) TRT configuration of the radar unit and the definition of the direct, reflected and scattered signals. (b) The GPR model where three $x$-polarized antennas are aligned in the $y$ direction

\section{The Radar Unit}

In a majority of GPR models found in the literature, the radar unit consists of a transmitter $(T)$ and a receiver $(R)[1,2,3]$. Then, the total signal collected by the receiver contains not only the desired scattered signal (S) due to the buried object, but also the direct signal (D), i.e., the incident field, due to the direct coupling from the transmitter to the receiver and the reflected signal $(G)$ due to the reflection from the ground. Usually, the D signa is much larger than the desired S signal, rendering the detection of the $S$ signal (and thus the buried object) difficult or impossible in the total received signal $(D+G+S)$, which is dominated by the $\mathrm{D}$ signal.

In this work, radar units, each consisting of two transmitters and a receiver, are modeled in transmitter-receiver-transmitter (TRT) configurations [4], as shown in Fig. 1(a). The receiver $(R)$ is located exactly in the middle of two identical transmitters $\left(T_{1}\right.$ and $\left.T_{2}\right)$, which are $180^{\circ}$ out of phase. In this configuration, the two direct signals $D_{1}$ and $D_{2}$ cancel each other at the receiver. Indeed, $D_{1}$ and $D_{2}$ cancel each other everywhere on a symmetry plane that is equidistant to the two transmitters. Similarly, the two reflected signals $G_{1}$ and $G_{2}$ also cancel out on the same symmetry plane and, in particular, at the receiver, if the ground is homogeneous and the ground-air interface is uniform. In that case, the total received signal becomes $S_{1}+S_{2}$, which is solely due to the buried object. 
Section III presents the simulation results obtained with such a GPR model, whose top view is shown in Fig. 1(b). The transmitter antennas are selected as small dipoles, each of which is modeled by a single Yee cube of constant current density in its volume. The time variation of the current source is given by

$$
J(t)=\frac{1}{\Delta^{3}}\left[4\left(\frac{t}{\tau}\right)^{3}-\left(\frac{t}{\tau}\right)^{4}\right] e^{-\frac{t}{\tau}},
$$

where $\tau=1 /\left(4 \pi f_{0}\right)$, and $f_{0}$ is the center frequency of the pulse, and $\Delta=\Delta x=\Delta y=\Delta z$ is the sampling interval in space.

The receiver is also modeled as a small dipole that samples and stores the values of the $x$ component of the electric field. Thus, discrete values of the electric-field function $E_{x}(x, y, z, t)$ are obtained at the receiver. When the radar unit is stationary and the receiver collects data at a point $\left(x_{0}, y_{0}, z_{0}\right)$ in space for successive instants of time, this is called an A-scan. A B-scan is obtained by performing repeated A-scan measurements at discrete points on a linear path. Similarly, a C-scan, which can be considered as combining several B-scans, denotes the data collected on a rectangular grid of discrete points on a constant $z$ plane.

\section{Simulation Results}

In this section, the GPR model introduced in Section II is used to simulate several scenarios. In all of these simulations, the center frequency of the excitation is selected to be $f_{0}=1 \mathrm{GHz}$. Sampling intervals in space and time are selected as $\Delta=2.5 \mathrm{~mm}$ and $\Delta t=4.5 \mathrm{ps}$, respectively, which satisfy the Courant stability condition. The transmitting and receiving antennas of the radar units shown in Fig. I(b) are separated by 2 cells. The relative permittivity of the ground is selected as $\epsilon_{r}=2$ unless otherwise stated.

\subsection{Conducting Prism}

The GPR model is first tested on a simple scenario: A perfectly conducting prism of $21 \times 21 \times 16$ cells is located 5 cells under the ground-air interface. B-scan data, shown in Fig. 2(a), is collected as it travels over the edge of the conducting prism $(y=11 \Delta)$ at a fixed elevation of 10 cells over the ground $(z=10 \Delta)$ and stops every $2 \Delta$ to perform an A-scan measurement. The maximum value of the electric field obtained in the B-scan is given in the title of the figure as an indication of the strength of the plotted signal. Careful investigation of Fig. 2(a) reveals that the GPR model responds only when the radar unit, is very close to the target, thus producing a localized response.

In order to further illustrate the response of the GPR model, the radar unit is moved on a two-dimensional grid, as opposed to a linear path. For each discrete radar position on the two-dimensional grid, an A-scan measurement is performed and the energy of the received $A$-scan signal is computed as

$$
\text { Energy }=\sum_{n}\left|E^{n}\right|^{2} .
$$

Figure 2(b) displays these energies on the two-dimensional grid obtained by the radar unit Figure 2(b) depicts that the radar unit obtains weak signals on the $y=0$ path, which coincides with the symmetry plane of the prism. However, this minima does not constitute an insurmountable detection problem, since two clear energy peaks exist on the two sides of this path. A complete C-scan or even a few B-scans would easily detect these energy peaks.

\subsection{Multiple Targets}

The previous section demonstrates that the GPR unit produces localized responses to nearby targets. The sensitivity of the radar unit to nearby targets can be beneficial for the detection of two closely buried objects. In order to investigate this situation, Fig. 3 presents the simulation results of a scenario, where two conducting prisms of $21 \times 21 \times 16$ cells are 
buried 5 cells under the ground, and separated by 20 cells. In Fig. 3 , the energies of the A-scan waveforms are evaluated according to Eq. (2) and presented in addition to the $\mathrm{B}$-scan results. Figure 3 shows that the GPR model produces signals that can be used for the detection of both objects.

In order to investigate the performance of the GPR model on two closely buried targets of different materials, two other simulations are performed. In Fig. 4(a), the scattering results of a cavity and a dielectric object with relative permittivities $\epsilon_{\text {cavity }}=1$ and $\epsilon_{\text {object }}=$ 8. respectively, are given. The two targets are buried 20 cells apart and 5 cells under the ground. which has a relative permittivity of $\epsilon_{\text {grond }}=4$. Figure 4(a) depicts that the GPR model clearly detects the two objects, even though the energy peak produced by the cavity is much smaller than that of Fig. 3(a) for a conducting object, and the energy produced by the dielectric object is even smaller. It is also observed that the waveforms reflected from the cavity and the dielectric object have their own characteristics. If the permittivity of the target is larger than the permittivity of the ground, the second reflected signal is stronger than the first, that is, the reflection from the lower face of the target is larger than that from the upper face. However, if the ground is denser than the target, then the reflection from the upper face of the target is stronger. This is due to the larger reflections encountered while passing from a denser medium to a rarer one, mainly caused by total internal reflections.

In the second simulation, the dielectric prism is replaced by a conducting prism. Figure 4 (b) shows that the objects are again visible, although the cavity is a weaker scatterer compared to the conducting object. Note that the same cavity is the stronger scatterer in Fig. 4(a) compared to the dielectric object.

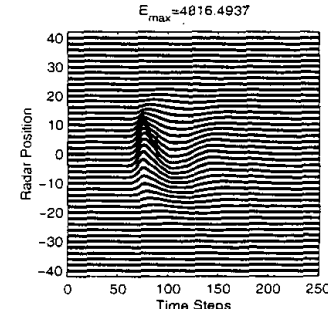

(a)

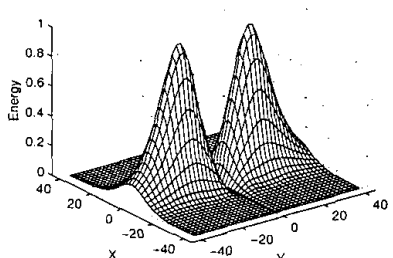

(b)

Figure 2: The simulation results of a perfectly conducting rectangular prism buried 5 cells under the ground. The ground model has a relative permittivity of $\epsilon_{\text {ground }}=2$. The radar unit travels on a linear path over the edge of the prism. (a) The B-scan results. (b) The energy diagrams

\section{Concluding Remarks}

The power and flexibility of the FDTD method are combined with the accuracy of the PML absorbing boundary conditions to simulate realistic GPR scenarios. Three-dimensional geometries containing models of radar units. buried objects and surrounding environments are simulated. In this paper, the radar unit is modeled as a TRT configuration. The buried objects are modeled as rectangular prisms with arbitrary conductivities and permittivities. Multiple-target scenarios are also simulated.

Using the simulation results, it is demonstrated that the major advantage of the TRT configuration is the total cancellation of the direct signals due to the direct coupling from the transmitters to the receiver and the partial cancellation of the reflected signals from the ground-air interface. Cancellation of these signals greatly facilitate the detection of the buried objects. 


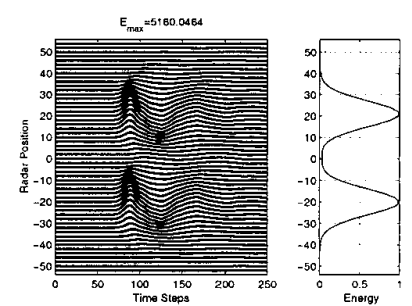

Figure 3: Two perfectly conducting prisms buried 5 cclls under the ground and separated by 20 cells.

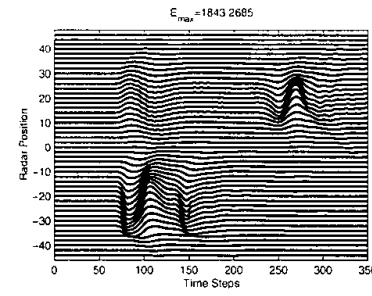

(a)

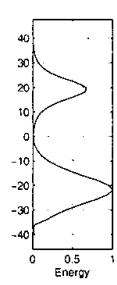

neroy

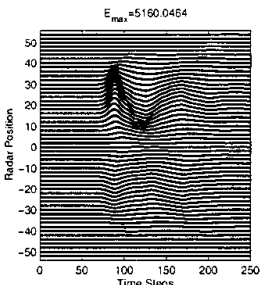

(b)

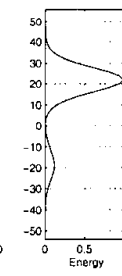

$-$

Figure 4: Two objects buried 5 cells under the ground and separated by 20 cells. The objects are (a) a cavity and a dielectric object with $\epsilon_{\text {object }}=8$, and (b) a cavity and a perfectly conducting object.

\section{References}

[1] M. Moghaddam. E. J. Yannakakis, W. C. Chew, and C. Randall, "Modeling of the subsurface iuterface radar," J. Electromagnetic Waves and Applications, vol. 5, no. 1, pp. 17-39, 1991.

[2] UT Oğu\% and L. Gürel. "Subsurface-scattering calculations via the 3D FDTD method employing P'YL ABC tor layered media," 1997 IEEE AP-S International Symposium and URSI Radio Science Meetrng, Montréal, Canada, July 1997.

[3] J. M. Bourgeois and G. S. Smith, "A complete electromagnetic simulation of the separatedaperture sensor for detecting buried land mines," IEEE Trans. Antennas Propagat., vol, 46, no. 10, pp. 1419-1426, Oct. 1998.

[4] P. Luneau and G. Y. Delisle, "Underground target probing using FDTD," 1996 IEEE AP-S International Symposium and URSI Radio Science Meeting, Baltimore, Maryland, U.S.A. pp. 1664-1667, July 1996.

[5] F. L. Teixeria, W. C. Chew, M. Straka, and M. L. Oristaglio, "Finite-difference time-domain simulation of ground-penetrating radar on dispersive, inhomogeneous, and conductive soils," IEEE Trans. Geoscience and Remote Sensing, vol. 36, no. 6, pp. 1928-1937, Nov. 1998.

[6] .J. P. Berenger, "A perfect]y matched layer for the absorption of electromagnetic waves," $J$. Comput. Phys., pp. 185-200, Oct. 1994.

[7] J. B. Verdu, R. Gillard, K. Moustadir, and J. Citerne, "An extersion of the PML technique to the FDTD analysis of multilayer planar circuits and antennas," Microwave and Optical Technology Letters, vol. 10, no. 6, pp. 323-327, Dec. 1995. 